\title{
Horizons/Théâtre
}

Revue d'études théâtrales

$14 \mid 2019$

Les arts du spectacle dans l'Afrique subsaharienne - 2

\section{Macabre et désordre ontologique dans l'œuvre théâtrale de Sony Labou Tansi}

Kedri Souâd

\section{OpenEdition}

1 Journals

Édition électronique

URL : https://journals.openedition.org/ht/1525

DOI : $10.4000 /$ ht. 1525

ISSN : 2678-5420

Éditeur

Presses universitaires de Bordeaux

Édition imprimée

Date de publication : 1 janvier 2019

Pagination : 74-91

ISBN : 979-10-300-0718-3

ISSN : 2261-4591

Référence électronique

Kedri Souâd, "Macabre et désordre ontologique dans l'œuvre théâtrale de Sony Labou Tansi », Horizons/Théâtre [En ligne], 14 | 2019, mis en ligne le 01 juin 2022, consulté le 30 juin 2022. URL

http://journals.openedition.org/ht/1525; DOI : https://doi.org/10.4000/ht.1525

\section{(c) $(7)$}

La revue Horizons/Théâtre est mise à disposition selon les termes de la Licence Creative Commons Attribution - Pas d'Utilisation Commerciale - Pas de Modification 4.0 International. 


\section{KEDRI SOUÂD}

Souâd Kedri est maître-assistante en langue et littérature françaises à l'université Mouloud Mammeri de Tizi-Ouzou. Actuellement, inscrite en thèse de doctorat en Sciences des textes littéraires, l'intitulé de la thèse : " Jeux et enjeux du macabre dans l'œuvre théâtrale de Sony Labou Tansi ». Intérêts de recherche : littérature du Sud, imagologie, approches de l'imaginaire, imaginaire de la mort, mythocritique, histoire du théâtre, esthétique et techniques du théâtre. Publications : «L'imaginaire de la mort dans l'État de siège d'Albert Camus », Revue d'Études Françaises, Université Eötvös Loránd à Budapest, 2014, Hongrie, http://cief.elte.hu/la-revue-detudes-francaises/editionspeciale-3/ souad-kedri ; "Les représentations stéréotypées de l'immigré africain dans Le Chercheur d'Afriques d'Henri Lopes ", Revue d'Études Françaises, n 21 , Université Eötvös Loránd à Budapest, 2016, Hongrie. http://cief.elte.hu/numero-21/numero-21/souad-kedri

Mail: souadkedri@yahoo.fr

Résumé : De Conscience de tracteur au Trou, l'écriture dramatique de Sony Labou Tansi questionne les grands problèmes de l'Afrique en mettant en évidence l'image des tyrans, de leurs victimes et du destin catastrophique de ce continent. Ce dramaturge révèle l'envers et l'endroit de l'Afrique à travers une symbolique de la mort globalement empruntée aux attitudes et aux pratiques funéraires traditionnelles négro-africaines. Le macabre traverse donc tout le théâtre de Sony Labou Tansi qui se décline au travers d'une philosophie négro-africaine. II est cependant symp-

Abstract : From Tractor Conscience to an Abyss, Sony Labou Tansi's dramatic writing questions the major problems of Africa by highlighting the image of tyrants, their victims and of the catastrophic fate of this continent. This playwright reveals the reverse side and the place of Africa through a symbolism of death globally borrowed from attitudes and traditional black African funeral practices. The macabre therefore crosses the whole theater by Sony Labou Tansi which comes through a Negro-African philosophy. It is however symptomatic that the objective of this article tomatique que l'objectif de cet article est de dégager du texte dramatique tansien, l'enjeu ontologique qui se présente comme des jeux rituels funéraires. De ce fait, nous solliciterons la notion de "rite funéraire ", selon la pensée de Louis-Vincent Thomas, pour voir comment le dramaturge congolais se saisit de cette pratique rituelle pour interpréter ce désordre ontologique qui révèle son caractère dévastateur sur l'ordre social.

Mots-cLÉs : macabre, rite funéraire, cadavres, zombies, désordre ontologique.

is to extract from the dramatic text of Tansin, the ontological issue that presents itself as funeral ritual games. Therefore, we will seek the notion of "funeral rite", according to the thought of Louis-Vincent Thomas, to see how the Congolese playwright grasp on this ritual practice to interpret this ontological disorder which reveals its devastating character on the social order.

KEYWORDS: macabre, funeral rite, corpses, zombies, ontological disorder. 


\section{Macabre et désordre ontologique dans l'œuvre théâtrale de Sony Labou Tansi}

Jacques Chevrier a Recensé dans son ouvrage, La littérature nègre, trois générations qui ont contribué à la conception d'un théâtre qui a puisé sa matière de l'histoire et de la réalité sociale et politique de l'Afrique noire. La première génération de dramaturges s'engage dans la lutte pour la liberté et conteste la politique coloniale de l'Afrique juste après les Indépendances. Cette génération s'inscrit dans la continuité du théâtre historique qui avait pour but de magnifier les héros du passé. Privilégiant la politique et l'histoire, Seydou Badian, Charles Nokan, Sidiki Bakaba et Sylavain Bamba ont dénoncé les conséquences du colonialisme, les mœurs politiques et les fractures sociales qui ont bouleversé l'Afrique subsaharienne. D’autres intellectuels engagés ont marqué les années 1960 : Bernard Dadié dans Monsieur Thôgôgnini, Les Termites d'Eugène Dervain, L'Homme qui tua le crocodile de Sylvain Bemba et Une saison au Congo d'Aimé Césaire. Ces œuvres dramatiques dénoncent une nouvelle réalité politique et sociale, conséquence de l'arrivée au pouvoir des héritiers de l'idéologie coloniale.

Pendant les années 1970, l’Afrique noire est fragilisée dans les domaines économique, politique et militaire. Par conséquent, l'engagement des romanciers et de dramaturges se poursuit en contestant la montée du despotisme instauré par les régimes armés. En résumé, l'évolution du théâtre en Afrique noire est rattachée au colonialisme, aux mœurs politiques et à la réalité sociale. Sony Labou Tansi a joué un rôle déterminant dans l'histoire du théâtre négro-africain. Il fait partie de ceux qui ont dénoncé avec virulence les potentats et leurs politiques. Il devient l'écrivain de la République populaire du Congo et la nouvelle référence négro-africaine de langue française. Un écrivain congolais, Henri Lopes, dit de Sony Labou Tansi : «Sony saisit le tison ardent et va semer le feu dans nos esprits et nos cœurs. Ce faisant il n'écrit pas pour l'histoire de notre littérature ni pour le critique. Il entame le dialogue avec tous. Cet enfant terrible réveille nos bonnes consciences assoupies et provoque les philosophes et les politiques ${ }^{1}$. $\gg$ Son talent de romancier, de poète, de metteur en scène et meneur de troupe s'est révélé à travers une littérature contestataire créée de 1974 à 1995. Ce penseur de théâtre, nourri de 
la verve nationaliste, a collaboré avec Nicolas Bissi pour fonder une compagnie de théâtre, le Rocado Zulu Théâtre à Brazzaville. La plupart de ses pièces théâtrales « dénoncent les comportements et les pratiques des hommes politiques qui n'arrivent pas à développer le pays et qui tuent tout le monde ${ }^{2} \gg$. À la lecture de son théâtre, nous remarquons que ce dramaturge théatralise cette situation chaotique en truffant ses pièces de symboles funéraires qui révèlent cette atmosphère macabre. Notre intérêt a été suscité par cette actualisation de cet imaginaire macabre dans son écriture dramatique. La mise en condition du macabre est structurée autour de plusieurs entités dramatiques, réduites à des cadavres et à des morts-vivats. Donc, nous nous poserons plusieurs questions : que révèlent ces symboles funéraires régis dans le texte dramatique tansien ? Peuvent-ils rendre compte des enjeux ontologiques du macabre ? Le rite funéraire est-il un moyen pour parvenir à l'ordre social dans le théâtre de Sony Labou Tansi?

\section{Scènes macabres et rites funéraires : vie et mort, un rapport de force}

Sony Labou Tansi offre aux lecteurs un théâtre satirique décuplé par des scènes macabres. Celles-ci dénoncent les maux des personnages tansiens et, par là même, de l'Afrique à travers une symbolique de la mort globalement empruntée aux pratiques funéraires traditionnelles. Sa prédilection pour les attitudes et pratiques traditionnelles négro-africaines est évidente dans ses œuvres romanesque et théâtrale. Ces pratiques sont insérées dans une esthétique dramatique à dimension grotesque et carnavalesque qui masque cet enjeu ontologique du macabre. De ce fait, nous avons remarqué que son théâtre est truffé de bout en bout de symboles funéraires ${ }^{3}$ qui viennent dépeindre le macabre qui « apparaît comme personnification de la mort. Ses modes de présence sont le meurtre ou action macabre, la dissolution ou décomposition cadavérique, la danse des morts, la situation macabre ou antagonisme de la Vie et de la Mort ${ }^{4}$. $\gg$ Notre analyse de cet imaginaire macabre se focalisera sur les symboles funéraires à commencer par le « cimetière », le « cercueil », les « cadavres » et les « deuilleurs ».

Conscience de tracteur s'ouvre sur une scène lugubre, « non loin du cimetière $^{5} \gg$, « une étrange cité souterraine ${ }^{6} \gg$ dans la ville de San-Mérina. Dans cette scène sous-titrée, « Le récitant », le dramaturge évoque une ville étrange qui vit au rythme d'un étrange vieillard, un personnage qui ne semble pas être ce qu'il est. Ainsi ce vieux s'adressant « aux travailleurs étrangement vêtus $^{7} \gg$ en ouvrant son livre noir, et dans une démarche désordonnée, il 
entreprend la lecture du « gros livre noir ${ }^{8} \gg$ qui fait le récit du chaos et de la fin du monde, car cette pièce est d'emblée politique au sens où plusieurs entités dramatiques sont en quête de solutions aux tourments de l'Afrique. Ce même personnage n'annonce pas seulement le chaos, mais le dramaturge lui confère un autre rôle, celui de père spirituel. Ce récitant annonce la cérémonie funéraire dédiée à Eva-Rivera et Ndola-Bambara en présence des officiels : le Général Leiso-Rava-Lata :

Au cimetière municipal de San-Mérina. Un monde fou. Au premier plan deux trous, deux cercueils, deux croix en or massif avec des inscriptions. Les cercueils sont recouverts $d u$ drapeau national. [...] On voit des larmes sur toutes les joues. Un prêtre avance. Il bénit les cercueils sans mot dire. On les descend au même. [...] La foule se disperse sans bruit. [...] Devant le palais. Le Général Leiso-Rava-Lata et les autres officiels rentrent du cimetière. On lit sur son visage la douleur qui creuse son cœur9.

Même rituel funéraire dédié à Mme Sawa et à Mme Aleluya :

Au cimetière de San-Mérina. Une foule assez nombreuse autour de deux cercueils. Un curé dit des prières. De temps à autre on entend un « amen $\gg$. On peut lire sur l'une des deux croix debout devant les bières: Madame Sawa, née LéhoMaté-1955-1995- et sur l'autre: Mme Aleluya née Sawa Lanza : 1972-1995.

On remarque le général civil. Il est songeur. On descend les deux cercueils au même moment.

Bruit de la terre sur les cercueils.

Quelques journalistes prennent des photos.

On chante un chant funèbre.

Puis l'on se disperse.

Un vent violent souffle sur le cimetière pendant longtemps.

Puis une forte pluie s'ensuit.

Après la pluie, tout le cimetière est envahi d'eau de ruissellement ${ }^{10}$.

Ces séquences ci-dessus mettent en exergue les étapes du rituel funéraire : le cimetière, la foule, le cercueil, le curé, le chant funèbre. Ces symboles sont significatifs des pratiques rituelles funéraires de l'Afrique noire. Cependant, 
à mesure que la pièce avance, nous remarquons que le dramaturge utilise le « cimetière », comme un espace qui illustre la violence, à des fins ontologiques. Pour preuve, Leiso et le fossoyeur sont à la recherche de la tombe d'Aleluya. Plus important encore, ils sont à la recherche de l'origine de ce désordre qui règne dans cet espace funèbre. Selon Leiso, la solution à ce désordre est dans la mort et dans la découverte de la tombe d'Aleluya, une militante attachée « à la valeur du sang humain ${ }^{11}$ » condamnée à être enterrée vivante : «J'ai souvent travaillé pour une chose, mon général : nous avons dépensé beaucoup de sang. Si j'accepte d'être enterrée vivante, c'est uniquement pour cela : savoir où va le sang que nous avons dépensé ${ }^{12}$. $\gg$

Dans Cercueil de luxe, nous avons relevé une autre cérémonie funéraire. L'action se déroule dans le quartier Radio-Baobab au pays de la République de l'Homme. Les villageois de Radio-Baobab habitent dans un cimetière, ils veillent un mort absent. Dans cette pièce, l'instrument funèbre est importé de l'Europe, il s'agit d'un « cercueil antiatomique », « une bière... un cercueil de première classe ${ }^{13} \gg$. Une foule qui veille un défunt absent dans un cimetière hanté par la folle Sofélina qui y habite depuis la mort de son mari. L'action se noue davantage avec l'arrivée de Paris du directeur technique aux Pompes funèbres, Christian Boucher. Dans cette scène, « tous tombent morts, puis reviennent à la vie ${ }^{14} \gg$. Ces villageois « morts-vivants » refusent la présence de Christian Boucher à la cérémonie de funérailles de Romain. Le chef du village qui a pour mission d'animer les cérémonies funèbres, ne parvient pas à animer la cérémonie du défunt, car Christian Boucher insiste à $\mathrm{l}^{\prime} \ll$ enterrer de manière scientifique ${ }^{15}$ » tout en ignorant les pratiques rituelles funéraires propres aux habitants du quartier Radio-Baobab :

Je suis peiné que vous l'ayez emballé comme de la viande de singe et que vous le pleuriez sans rime ni raison, avec vos larmes sans tête ni queue. (Lundi ${ }^{16}$ veut toucher le cercueil) Attention, monsieur : ne voyez-vous donc pas comment vos mains en sont indignes? Cet alliage spécial ne peut être touché qu'avec des gants spéciaux ${ }^{17}$.

On peut constater que Christian Boucher ignore l'importance de ces pratiques rituelles traditionnelles de l'Afrique noire. Louis-Vincent Thomas soutient justement que la cérémonie funéraire est une forme indispensable pour renforcer la cohésion sociale et valoriser les pratiques traditionnelles. En anthropologue de la mort, Sony Labou Tansi a subverti cette pratique en incorporant dans la scène un personnage qui représente l'Europe et qui vient contaminer l'ordre social. En effet, ce désordre social se manifeste dans une scène macabre et tragique où Mercredi, le vieux paysan, témoigne de la 
situation chaotique de son pays : « Le pays a changé. Ses habitudes boitent. Nous ne sommes plus nous. Nous ne serons plus jamais nous : quand même! Le marmot nous fait marcher. Il nous marche dessus ; il marche sur nos cœurs ! ${ }^{18} \gg$ Dans cette scène, Christian Boucher est l'homme du désordre. Sans prendre le soin de justifier ce rite de passage, le chef du village condamne Christian Boucher à la pendaison : «N'ayez aucune crainte, fiston : c'est la seule marque de mort pour laquelle nous n'avons besoin ni d'assistance technique, ni de transfert de technologie. Nous pendons sans douleur depuis la nuit des temps ${ }^{19}$. $\gg$ Ce faisant, les villageois de Radio-Baobab manifestent leur puissance en condamnant Christian Boucher à la pendaison. Le lieu de la condamnation est le « cimetière ». Dans cette pièce, cet espace funéraire fait corps avec le désordre et l'ordre. En termes plus explicites, le « cimetière » est le lieu du désordre social, car Christian Boucher s'est attaqué à une pratique traditionnelle en tant que fait culturel ; et le lieu de l'ordre social, grâce à l'intervention de la folle Sofélina qui supplie Romain, ce fils occidentalisé, de se réconcilier aves ses pères, ses traditions, et par conséquent, son « Afrique »:

Sofélina. - Voici trois mois que tu es dans cette condition de bête de boue. Je te prie, ils ne t'en demandent pas trop: danse leurs onze danses et je suis sûre que tu seras guéri.

Romain.- La mort m'est plus douce la soumission.

Sofélina.- Il y a des hommes qu'aucune mort ne peut atteindre. Je t'en prie : danse leurs onze danses. Fais-le pour ton cour et pour moi.

Romain.- Tu deviens subitement la femme, et pour toi, j'ai maintenant envie de la vie. Moi l'ancien mort veux vivre : va leur dire que je suis prêt à danser leurs danses quand ils voudront ${ }^{20}$.

Dans cette séquence, le dramaturge met en scène le caractère tenace du personnage de Sofélina qui incarne la réconciliation. Cette entité dramatique macabre a accompli une mission messianique en inculquant à Romain la notion de la réconciliation. Le « cimetière » devient donc un espace de l'ordre social.

Par ailleurs, nous avons remarqué que le rite funéraire n'est pas continu dans toutes les pièces théâtrales de Sony Labou Tansi. Expliquons. Dans Qu'ils le disent, qu'elles le beuglent, l'espace funèbre est réduit à la mer. C'est à la mer que les corps sont jetés : «Le jour se lève. Nous rêvons plus tard. Commençons par le commencement des choses, nettoyons. À la mer, les corps ! Parmi les naufragés encore en vie. L'accident sera parfait ${ }^{21}$. $\gg$ Dans Qui a mangé Madame d'Avoine Bergotha ? l'espace funèbre est la province de 
Bergotha, le « pays des morts ${ }^{22} \gg$, une île créée par Walante, un tyran qui « s'apprête à égorger tous les mâles de l'île ${ }^{23}$. » Dans cette pièce, ce tyran a pour projet lugubre d'exécuter tous les mâles de Bergotha et de se proclamer unique géniteur pour peupler l'île. Pour ce faire, il fait appel aux inséminateurs. L'extrait suivant en est une illustration :

Walante: [...] Voici l'article unique de notre constitution :

« Les hommes sont démis de leurs fonctions en tant que peuple.

Moi, Walante, je les remplacerai, personnellement,

dans toutes leurs fonctions.

Moi, Walante, je me programme et me planifie

avenir

espoir

et volonté personnelle du peuple!

Fondateur de son existence,

animateur de ses raisons,

gérant et garant de ses consciences,

son inséminateur unique,

commandant de sa force de vie $e^{24}$.

Ce tyran anglais est très épris de Madame d'Avoine Bergotha, ce personnage dramatique est un homme travesti qui vient « du fond de la mort ${ }^{25}$. » Dans cette pièce, des scènes macabres crues et accrues révèlent l'horreur de l'extrême violence que les figures morbides exercent sur les personnages « vivants », « morts » et « morts-vivants ». Cette horreur est décrite à la fin de la «scène-thé-citron » en ces termes : «Dans la forêt, chasse à l'homme, aboiements de hordes de chiens renifleurs, tintamarre, casses, fusillades interminables, appels du cor, hurlements ${ }^{26}$. $\gg$ À mesure que la pièce avance, les morts victimes de la répression de Walante s'accumulent : «Madison : C'est étrange de dire ses amours quand tout s'écroule. Que viens-tu aimer sur cette colline de cadavres crus ? ${ }^{27} \gg$ Nous avons donc constaté que ces morts n'ont pas eu le droit à la cérémonie de leurs funérailles. Ces suppliciés sont pourchassés et menacés par des morts-vivants. Autrement dit, ces suppliciés deviennent les victimes de zombies. À travers la symbolique de ces figures morbides, le dramaturge veut banaliser le rite funéraire pour révéler cette banalisation de l'horreur et de la violence, et par là même, de la vie. Ici, le dramaturge délaisse la dimension rituelle funéraire du mort, un rite important dans les traditions culturelles africaines, pour masquer ce désordre social. Cette vision apparaît aussi dans la réplique du récitant dans Conscience de tracteur : « [... N N'oubliez pas, entre la survie de l'homme et ses idées, il n'y a ni équivoque, ni compromis, entre la survie de l'homme et ses traditions, on ne peut 
sacrifier que ses traditions ${ }^{28}$. $\gg$ Ainsi, ce désordre ontologique est révélé au niveau de l'espace funèbre dans lequel évoluent les vivants, les morts et les morts-vivants. Par le biais du cérémonial des initiations les plus animistes, ce dramaturge congolais convoque toute une symbolique de la mort, car il subvertit quelques éléments du rituel funéraire, non pas pour réconcilier pratiques rituelles africaines et pratiques occidentales, mais pour que ce travail de déconstruction au niveau numineux, lui permet de dénoncer ce désordre social catalyseur du désordre ontologique.

Dans La Parenthèse de sang, les personnages tansiens sont ancrés dans un univers lugubre, déshumanisé et gagné par la peur et la terreur. Les soldats sont à la quête d'un mort, d'un révolutionnaire assassiné, d'un résistant. Le pouvoir ne croit pas à sa mort : « Le Sergent. - J'en ai par-dessus le c... Non. Qu'on tire ça au clair avant qu'on ne s'affole tous. Tout un pays de fous. Cette tête, qu'on l'emmène. Je crois que cette fois la capitale finira par comprendre QUE LIBERTASHIO EST MORT ${ }^{29}$. $\gg$ Ces soldats et sergents persécutent Martial-Makaya, neveu de Libertashio, Kalahashio, femme de Libertashio et leurs trois filles, Ramana, Aleyo et Yavilla, car ils sont condamnés par le pouvoir local. Dans cette pièce, le lieu de la mort et de l'exécution de ces condamnés est le tombeau de Libertashio. Ainsi, la tombe de Libertashio est le lieu de l'exécution, un espace funèbre destiné à la mort. Comme on le sait, la tombe représente la « pérennité de la vie, à travers ses transformations ${ }^{30}$ ». Dans les traditions africaines, ce symbole funéraire est la demeure de l'âme du mort, pour empêcher que les âmes vagabondes ne viennent tourmenter les vivants. En somme, ce sont les morts qui témoignent de la mort, en sursis, des vivants au milieu d'un tombeau. Mais d'un tombeau vide, car l'âme de Libertashio vagabonde dans le cimetière. Cette errance de l'âme de Libertashio est probablement liée à l'absence de l'un des principaux procédés du rituel funéraire, «l'accumulation des rites aux moments cruciaux du devenir du défunt $t^{31} \gg$. En effet, Libertashio est symboliquement privé de « secondes funérailles » qui « constituent un rite qui désolidarise les survivants du défunt (fin du deuil) et permet à ce dernier d'accéder à son nouvel état, celui d'ancêtre par exemple $\mathrm{e}^{32} \gg$. Plus important encore, ce rite a pour fonction de «propitier l'âme ${ }^{33} \gg$ en fixant l'âme du défunt pour empêcher son errance :

Le Fou. - Hé ! S'il vous plaît! Pas de sang sur la tombe ! Pas de sang! [...]

Cavasha, dehors. - Les condamnés seront fusillés sur la prétendue tombe de Libertashio. » 
Aleyo, Le Docteur, Yavilla, Ramana, Martial, Kalahashio.- Ils n'ont pas encore tire $^{34}$.

On peut constater que cette absence de ce procédé rituel révèle cette déchéance des valeurs sociales. Or Louis-Vincent Thomas atteste $\ll[\ldots]$ que le rituel funéraire manifeste précisément l'intention de neutraliser le désordre dont la mort est le signe et de canaliser la régénérescence qu'elle implique ${ }^{35} \gg$. Plus important encore, Libertashio envisage de faire de cette contrée de la mort qu'est l'Afrique, une contrée de la vie, mais il va falloir sacrifier quelques traditions pour rendre la dignité à l'homme, à la mort, et donc, à la vie. LouisVincent Thomas note justement :

[...] dans les sociétés traditionnelles, il s'agit de ritualiser la rupture qu'est la mort pour la rendre supportable. Liturgie parce que comportement hautement symbolique, thérapie parce que codification du chagrin, règles normatives afin de mieux préparer le mort à son nouveau destin, les funérailles apparaissent avant tout comme un rituel d'adieu. C'est dans et par les funérailles que le mort n'en finit pas de mourir ${ }^{36}$.

Dans Qu'ils le disent, qu'elles le beuglent, Une chouette petite vie bien osée, Monologue d'or et noces d'argent, Une vie en arbre et chars... bonds, La Peau cassée, Le Trou, les cadavres animés côtoient les « vivants », mais cette entité dramatique macabre est privée de rite d'initiation. Ces cadavres évoluent dans un espace morbide qui ne reflète que désordre et marasme. D’ailleurs, nous avons remarqué que le dramaturge a subverti un autre procédé du rituel funéraire, il s'agit au du rassemblement des vivants pour inhumer un mort. En effet, dans Cercueil de luxe, les morts-vivants se sont rassemblés au cimetière de Radio-Baobab, sous l'ordre du chef spirituel, pour inhumer un mort-vivant, Romain. Dans ces pièces, l'Afrique est donc représentée comme un cimetière à ciel ouvert où sont enterrés les morts sans rituel funéraire. $\mathrm{Ne}$ peut-on pas dire que le dramaturge tente de dévoiler cet effondrement des valeurs traditionnelles écrasées sous le poids de la violence, de la terreur et de la honte. Le choix des cadavres ou des morts n'est, d'ailleurs, pas anodin. Ils sont longuement évoqués dans son théâtre.

En mettant en concurrence ces symboles funéraires, le dramaturge dénonce cette déchéance des traditions africaines les plus animistes, contaminées par le désordre social, depuis l'émergence des dictatures après les Indépendances. Pour ce faire, le dramaturge, et en tenant compte du piment de l'horreur, il a su incorporer dans le discours des personnages, leur impuissance face à la violence, à la vie. À l'exemple de la violence psychologique qu'exerce l'ombre de Libertashio sur les soldats, et donc, sur ses bourreaux. Ces soldats sont hor- 
rifiés par l'image spectrale d'un rebelle qui menace l'ordre social. À travers le grotesque, le dramaturge s'attaque aux agissements d'hommes politiques africains. En somme, Sony Labou Tansi veut dénoncer cette violence qui bouleverse l'ordre social, et qui vient même détruire les valeurs traditionnelles. On peut constater que le dramaturge subvertit quelques pratiques rituelles pour non seulement défier une certaine classe d'hommes politiques africains, mais aussi pour insister sur cette quête intermittente de l'espoir, de la paix et de la vie. Pour preuve, Louis-Vincent Thomas atteste que « les actes rituels ont un effet cathartique en tant qu'expression libératoire des angoisses et mode de résolution des drames et conflits ${ }^{37} \gg$.

\section{Cavavres et zombies. Entités dramatiques macabres}

Ce qui contribue au renforcement du macabre dans le théâtre de Sony Labou Tansi est cette prolifération d'entités dramatiques morbides, à plusieurs facettes, qui témoignent de l'extrême violence d'un pouvoir politique responsable de la déchéance sociale et économique, et donc, du chaos de l'Afrique. En effet, la plupart des pièces théâtrales sonyennes présente plusieurs figures déshumanisées qui nourrissent l'intrigue et le ridicule. Ainsi, ce dramaturge de talent fait « fonctionner » les morts en donnant la parole aux cadavres qui se confrontent et s'entremêlent. Ceux-ci se logent dans des espaces funèbres. Plus important encore, ils se déploient dans des scènes macabres en fonction de symboles funéraires insérés dans le texte dramatique. Ils sont éparpillés dans les dialogues, les chœurs et les didascalies, et ils n'apparaissent pas énigmatiques. En effet, Sony Labou Tansi a conféré une identité à chaque cadavre en transgressant les limites de la réalité. Généralement, le cadavre représente un corps sans vie, inanimé, mais dans le théâtre sonyen, il est animé. Ce dramaturge met, ainsi, à sa disposition plusieurs figures par lesquelles il réussit à rendre vraisemblable la violence des scènes macabres. Interrogeons-nous sur ces cadavres et zombies.

Comme on l'a déjà dit plus haut, Sony Labou Tansi a utilisé les morts sous les traits de cadavres et de zombies. Ces entités dramatiques semblent manier avec assurance le meurtre. Ces «agents » macabres manifestent une violence qui engendrent massacres et folies meurtrières. Dans Le trou, le lieutenant Nouany est assassiné $: \ll[\ldots]$ On a retrouvé sa tête à l'entrée du camp Banardy. Le reste du corps a disparu ${ }^{38}$. $\gg$ Une autre scène macabre représentative de l'hostilité de ces zombies dans Conscience de tracteur:

Le récitant: Devant le colonel et le commissaire, passe le peloton; derrière eux, on traîne des prisonniers déchiqueté, saignant frais. 
Eva : C'est comme dans songe très sale. Tout prend la couleur du sang. [...] Je pense... à ce petit voyou qu'on a descendu hier au marché de la Plata. Un vrai mignon. Quinze ans... peut-être. Ça voulait résister. Et comme tout le monde est déjà dépassé, comme tout le monde est déjà fou, on a planté quatre chargeurs P.M. dans son cour. Il s'est effondré, mais il ne crevait pas. On a doublé la ration, même résultat. J'ai donné l'ordre de laisser tomber. Comme j'allais partir, il a saisi fermement le pied de mon pantalon. Puis, la bouche blonde de sang, il a dit : «Commissaire! 》 La même petite phrase satanique : «Commissair» - ce que vous faites avec du sang... Ça m'a mis hors de moi. Je lui ai foutu un coup de pied dans les yeux. Il a saisi la pointe de mon pantalon entre les dents. Pour m'en tirer, il a fallu que je coupe, qui je lui laisse un bout de mon pantalon. Puis il a crevée ${ }^{39}$.

Dans une autre pièce, La Peau cassée, le dramaturge met en scène la folie meurtrière d'Alvaro Sanza qui prend l'apparence d'un tueur. Celui-ci est obsédé par la volonté de se venger de Bunglstone : «J’ai quand même mis huit ans à construire la mort de Bunglstone ${ }^{40}$. $\gg$ Dans La Parenthèse de sang, les scènes de violence se succèdent d'une façon effrénée. À la fin du Deuxième soir, Martial et Cavacha, deux condamnés à mort, qui se posent une question existentielle : quelle mort mourir ? Le passage ci-dessous en est une illustration :

Cavacha, il grince des dents. On devrait vous condamner à trente morts.

Martial. - Il ne fut créé mort, hélas! Une en deux : celle des hommes et celle des rats.

Cavacha, furieux. - Tu auras quinze doses de ta mort à toi. Quinze doses. Tu mourras quinze fois...

Martial. -Ça ne fera qu'une mort, hélas !

Cavacha. - Une mort en quinze étapes. Une mort multipliée par quinze.

Martial.- Égale une mort.

Cavacha, aux soldats.- Coupez-lui la main droite. Crevez-lui l'œil droit. Évitez les pertes inutiles de sang. Il faut qu'il meure par région. Coupez les oreilles, coupez le nez.

CRIS DE MARTIAL.- Le temps de mourir est passé. Le temps de mourir est passé... Passé... Passé... Passé $e^{41}$.

On peut constater que ces passages révèlent les armes de la satire utilisées par le dramaturge pour montrer cette banalisation de la vie humaine à travers les agissements de ces zombies. Ainsi, cette folie criminelle est une façon de 
dévoiler d'innombrables facettes déshumanisantes des potentats. De surcroit, ces «agents » macabres émaillent Qui a mangé Madame d'Avoie Bergotha? Leur folie meurtrière est inéluctable à travers des figures de cadavres animés et errants qui répugnent :

Walante : Ça bande infernalement dans mon crâne.

Sujets riches, pauvres, grands, petits, tordus, truands, génies, nous sommes vêtus de cette ample tristesse Nous nous esquintons à vouloir éteindre la lumière! C'est là un métier déprimant, je vous jure!

Comme il n'est plus rien de noble à faire, sur votre terre pourrie, je fabrique la trouille qui boit les âmes sans pitié, les âmes et les consciences! Je gouverne une nation dissoute, Je règne sur un peuple dissout! Ah comme c'est beau le métier de vaincu.

Un homme de mon calibre, sachez cette chose, ne pleurniche pas sur ses choix!

(À Yongo-Loutard) Enfermez la marmaille!

Qu'ils se saoulent de bonnes nouvelles pendant seize nuits!

Ensuite, je les pendrai... puis les guillotinerai.

Je me sens un animal essentiel.

Mon caur enfle, mes nerfs mordent à mes os!

Je les guillotinerai pour mauvaise collaboration avec le diable ${ }^{42}$.

Dans Qu'ils le disent, qu'elles le beuglent, les corps sont jetés à la mer de Belmonta, le « pays de la mort ${ }^{43}$ » Dans cette pièce, le dramaturge donne la parole à un «mort-vivant » pour briser le silence. Ainsi Zooam témoigne de l'extrême violence du régime dictatorial de l'Afrique moderne :

La détresse des autres ne nous regarde pas. Une barque. Elle est bondée... Elle vient. La barque est remplie de femmes, de paysans, de moines... Je ses en moi un ardent désir de dormir. Mais où que je veuille fermer les yeux, les cornes saillantes de notre passé me regardent. Elles remplissent ma personne d'un vibrato sourd et froid, une ardente langueur, un fou désir d'embrasser le vide... C'est tragique de réaliser que toute notre vie durant, nous n'avons fait que bricoler la mort ${ }^{44}$. 
Effectivement, une autre exécution sommaire est mise en scène dans une fable dramatique Une chouette petite vie bien osée, où des «zombies» font le malheur des « vivants » et des cadavres. Ceux-ci sont jetés d'un hélicoptère :

Coppola : [...] Je t'avertis, Esther, nous sommes arrivés dans un pays qui a rang de mouroir : pas de café, pas de sucre, pas de beurre, pas de dessert. Les seules choses qu'on trouve ici, c'est l'appel à l'immatriculation quotidienne, l'enregistrement et le marquage scientifique du peuple. $[\ldots]$

Emanu : Il en meurt trop. Il faut maintenant que quelqu'un arrête cette jactance de vouloir faire pondre de l'eau à ces pierres en fournaise.

Mikael: [...] C'est devenu un culte pour ces bougres. Mais je les comprends tout à fait : nous végétons dans cette poussière aride depuis douze ans déjà. Il fallait inventer un prétexte de vie. [...] L'armée que nous fûmes est, à ce jour, réduite à fusiller de la vermille, dans cette manière de ruine fauve, saignante, dure. Nous sommes là, entassés, tels des cadavres, à trembler et à craindre des morsures de sales bêtes. Sans eau, sans pitance et sans médicament. Sans tabac, sans café. Piétinés par ce temps merdeux, nous sommes là en train de gérer une enfoirade sans nom ${ }^{45}$.

Comme dans les pièces précédentes, le dramaturge donne la parole aux morts et réduit au silence les vivants pour dépeindre irrémédiablement du destin catastrophique de l'Afrique.

Dans une autre pièce, Monologue d'or et noces d'argent, Sony Labou Tansi donne la parole à une foule de zombies qui hante un village cimetière visité par l'Homme-Monstre, son Homme-Montre, ses géniteurs d'emplois et ses taureaux chicoteurs. Carmanio, un village cimetière où se logent les voix des morts enregistrées par le mambarinier, l'arbre de Gohomsaya qui interroge la mort et ses vies. Le vieillard, les deux femmes, l'enfant et Mensfield, des entités dramatiques «zombies », torturés et se seraient vu condamnés au bûcher pour : « refus d'obtempérer, incitation au fanatisme collectif, et à la fainéantise tribale, rébellion ouverte, outrage au progrès, crime contre l'humanité, entrave délibérée à la marche en avant du monde, pratique préméditée de l'obscurantisme et de la sorcellerie athée, recours à l'alchimie et à la sauvagerie $^{46}$. »

Ainsi, il juxtapose, grâce au désordre social révélé dans des espaces funèbres, ce désordre ontologique, et il en fait un point exact de l'équilibre entre les vivants et les morts. Expliquons. Face à cet univers morbide, la passion criminelle des zombies n'est pas une fin en soi. À cette catégorie de zombies hostiles s'oppose celle qui incarne l'ordre, la paix et la réconciliation. Dans 
Conscience de tracteur, Le Vieux est figuré sous les traits d'une entité dramatique macabre de type «messianique »: «Le Vieux - Ah, vous ne saisissez point dans toute leur plénitude les dires d'un mort qui flotte dans la vie ${ }^{47}$. » Ce vieux au pouvoir messianique relate dans une longue tirade les épreuves d'un autre Déluge en invoquant le « commencement » :

Depuis quinze ans, je me suis mis à construire ce que j'ai tendrement baptisé la Nouvelle Arche de Noé. Oh, un job assassinant. [...] J'ai rassemblé dans mon arche près de quatre mille individus et plus de douze mille espèces de plantes et d'animaux. Restait seulement à déclencher le déluge, un déluge de lumière. Dans dix jours la terre ne serait plus qu'un triste amoncellement de carbone, car dans dix jours devait éclater ce que j'appelle la bombe carbonique. Puis pendant neuf ans, il n'y aurait plus de jours, il n'y aurait plus de nuits. [... Il n'y aurait plus que du temps, posé sur les mondes comme un peu de poussière. Puis au début de la dixième année la nouvelle humanité sortirait lentement de sa chrysalide. [...] Ce serait donc des hommes, guéris de leur raison perverse, de leur Histoire nauséabonde et de leur ancien comportement. [...] Et la terre ne serait plus qu'une longue chanson, un long refrain d'amour et de paix, un immense jardin de rêves. J'attendais avec impatience le jour où je devais déclencher cet ordre de choses $^{48}$.

À partir de cette longue séquence sus-citée, nous remarquons que le dramaturge a inséré dans le discours de son personnage les éléments essentiels d'un récit mythique, celui du Déluge. Ainsi, il mobilise les forces de ce « zombie » pour transmettre un message d'espoir en rendant à l'homme sa dignité. À côté de cette pièce, La Peau cassée, Line est une entité dramatique zombie qui souffre de sa condition de cadavre : « Je n'arrive pas à briser le cadavre insipide où vous m'avez enfermée, mon père et toi. Tu voulais me posséder comme une pierre qui brille. Pour tes yeux et pour tes désirs ${ }^{49}$. $\gg$ Alvaro Sanza projette de massacrer Bunglstone pour sauver les « damnés de la terre ${ }^{50} \gg$, les «trompés de la terre ${ }^{51} »$, Line, Laure et Daniel :

Line. - [...] Recherchons la lumière qui peut-être est venue se cacher dans cette forêt opulente. Et comme tu me disais, si le corps n'est qu'une adorable naïveté, pourquoi ne pas apprendre à exiger cette lumière étrange qui brille dans les choses. [...] Ce que j'aime d'Alvaro, ce que j'en ai toujours aimé, c'est cette étrangeté : la lumière qui a toujours inondé son cil. La paix qu'il a fait pour la haine, Alvaro, tous les gestes, la lumière qui brille dans tes yeux, ta façon de marcher, ta manière de respirer, tout en toi exige et commande la paix. (Daniel reprend la hache) Tout ton corps appelle à la lumière et à l'amour. Et mon corps à moi attend $d^{52}$. 
Il n'est, à cet égard, pas innocent, que de nombreuses tirades soient énoncées par des morts-vivants qui sèment la terreur « au pays des morts ${ }^{53}$ ». Nous pouvons dire dans ce cas que les morts représentent le désordre social et les morts-vivants, le désordre ontologique, pour parvenir à l'ordre social. Ces zombies incarnent donc cette figure de médiateur « vie-mort » qui a pour fonction d'expliquer ce désordre, et par là-même, d'établir un ordre social. En somme, cette entité dramatique macabre figure le chaos pour défendre l'homme, la vie et parvenir à l'ordre social. En définitive, nous pourrions dire que le choix de cadavres et de zombies oriente la vision de Sony Labou Tansi sur ce désordre ontologique que veut ce dramaturge dévoiler à travers ces «agents » du macabre qui jouent le rôle de «médiateur » entre la vie et la mort. Ces entités dramatiques macabres contestent ce désordre social qui reflète immanquablement ce désordre ontologique. Ainsi, Sony Labou Tansi sacrifie les traditions négro-africaines pour rendre la dignité aux hommes et aux femmes comme le dit si bien Le récitant dans Conscience de tracteur : « [...] N'oubliez pas, entre la survie de l'homme et ses idées, il n’y a ni équivoque, ni compromis, entre la survie de l'homme et ses traditions, on ne peut sacrifier que ses traditions ${ }^{54}$. »

En définitive, ce dramaturge fait «fonctionner » les vivants et les morts pour subvertir les traditions les plus animistes qui font la force et la puissance de l'Afrique afin de masquer son projet humaniste. En somme, le théâtre de Sony Labou Tansi offre une mise en condition cérémoniale du mort et du mort-vivant qui connaissent une autre mort. Ces entités dramatiques macabres pensent comme des philosophes de la mort. De ce fait, ils sont arrivés au zénith de la réflexion métaphysique humaine. Pour preuve, dans le prologue du troisième soir Ramana dit : «Y a peut-être des morts que la vie poursuit jusqu'à la mort ${ }^{55}$. $\gg$ En ce sens, l'homme se trouve dans l'impossibilité d'oublier la mort, la solution serait dans la vie, la réconciliation et le pardon, donc, le recommencement. Alors, ne peut-on pas dire qu'il met en spectacle des entités dramatiques «zombies» de type «messianique » pour transcender le désespoir?

Pour conclure, Sony Labou Tansi a créé un théâtre de la « vie-mort » où symboles funéraires, cadavres et zombies sont contaminés par le désordre social dépeint par le dramaturge dans son texte dramatique. La visée de Sony Labou Tansi est claire : déshumaniser l'homme pour universaliser la paix et l'espoir. En effet, en plaçant le macabre au cœur de son théâtre, ce dramaturge de talent dépeint cette obsession du mourir doublée de cette volonté de vivre. En somme, le sens du macabre dans l'imaginaire de la mort de Sony Labou Tansi peut être saisi grâce aux pratiques rituelles funéraires subverties et dé- 
construites, incorporées dans une esthétique dramaturgique éclatée. Il y a effectivement des éléments de la diégèse qui confirment que ces rites funéraires à travers l'omniprésence des morts, viennent accroître l'enjeu ontologique du macabre. Les pièces théâtrales de Sony Labou Tansi contiennent donc des rites funéraires non pas pour dépeindre $\ll$ cette mort de la vie ${ }^{56} \gg$, mais pour révéler des valeurs existentielles qui pourraient éventuellement guider l'Africain, et donc, l'Homme. Comme le signale Louis-Vincent Thomas : «Démystifier la mort, ses pompes et ses œuvres peut aider à mieux comprendre le sens de la vie ${ }^{57}$. $\gg$ Certes dans l'écriture dramatique sonnyenne, le macabre est une expression du morbide, du désespoir et de la déshumanisation, mais il est aussi l'expression de la vie, de l'espoir et de l'humanisation.

\section{Bibliographie}

Chevalier Jean, Gheerbrant Alain, Dictionnaire des symboles, Paris, Robert Laffont et Jupiter, 1982.

Chevrier Jacques, Littérature africaine, Paris, Armand Colin/HER, 1999.

— Conscience de tracteur, D.A.E.C. Coopération, 1973.

—, La Parenthèse de sang. Je, soussigné cardiaque, Paris, Hatier International, 1981.

—_, Qui a mangé Madame D'Avoine Bergotha ?, Lansman, 1989.

—, Une chouette petite vie bien osée, Lansman, 1992.

- Une vie en arbre et chars... bonds, Lansman, 1992.

—_, Qu'ils se disent, qu'elles le beuglent, Éd. Carnières-Morlanwelz, Éd. Lansman, 1995.

— Monologue d'or et noces d'argent, Le trou, Éd. Carnières-Morlanwelz, Éd. Lansman, 1995.

- Cercueil de luxe. La peau cassée, Éd. Montreuil-sous-Bois, Éditions Théâtrales, 2006.

—, La Chair et l'Idée, Éditions Les Solitaires Intempestifs, 2015.

M'Bemba-Ndoumba Gaston, Un coup de théâtre. Histoire du théâtre congolais, Paris, L'Harmattan, 2008.

Thomas Louis-Vincent, Anthropologie de la mort, Paris, Payot, 1980.

—_ Cinq essais sur la mort africaine, Éditions Karthala, 2013.

—, La mort africaine, Paris, Payot, 1982. 1975 .

— Le cadavre, Bruxelles, Éditions Complexe, 1980.

- Mort et pouvoir, Paris, Payot, 1978.

- Rites de mort, Fayard, 1985. 


\section{Notes}

1. Sony Labou Tansi, La Chair et l'Idée, Éditions Les Solitaires Intempestifs, 2015, p. 299.

2. M'Bemba-Ndoumba Gaston, Un coup de théâtre. Histoire du théâtre congolais, Paris, L'Harmattan, 2008, p. 31.

3. Louis-Vincent Thomas explique dans Anthropologie de la mort que « [...] l'analyse du rituel, initiatique ou funéraire nous semble particulièrement précieuse car elle souligne en quoi le symbole permet de dépasser la contradiction mort/naissance. [...] Mise à mort symbolique du néophyte dans l'initiation, rituel symbolique, rituel symbolique d'échange dans les funérailles et les rites post mortem vérifient sans équivoque en quoi les diverses formes et forces sociales sont imprimés par des acteurs selon un double registre : celui de l'expression qui, par le recours au code symbolique, impulse une longue et complexe chaîne de signifiants et celui de l'interprétation qui, par le biais du maître de rituel, proscrit le désordre. En effet, de même qu'en mimant la mort (biologique) le rite initiatique se fait créateur de vie (sociale), de même en jouant les conflits ou les tensions, il permet de les sublimer. » Paris, Payot, 1980, p. 114.

4. Ibid., p. 158.

5. Sony Labou Tansi, Conscience de tracteur, D.A.E.C. Coopération, 1973, p. 8.

6. Ibid., p. 8.

7. Ibid., p. 8 .

8. Ibid., p. 8.

9. Ibid., p. 52-53.

10. Ibid., p. 69.

11. Ibid., p. 68.

12. Ibid., p. 68.

13. Sony Labou Tansi, Cercueil de luxe. La peau cassée, Montreuil-sous-Bois, Éditions Théâtrales, 2006, p. 15.

14. Ibid., p. 15.

15. Ibid., p. 14.

16. Chef de village, chef spirituel après le curé.

17. Ibid., p. 14.

18. Ibid., p. 11.

19. Ibid., p. 16.

20. Ibid., p. 22.

21. Sony Labou Tansi, Éd. Carnières-Morlanwelz, Éd. Lansman, 1995, p. 28.

22. Sony Labou Tansi, Qui a mangé Madame D’Avoine Bergotha? Lansman, 1989, p. 102.

23. Ibid., p. 43.

24. Ibid., p. 52-53.

25. Ibid., p. 71.

26. Ibid., p. 65.

27. Ibid., p. 69.

28. Sony Labou Tansi, D.A.E.C. Coopérattion, p. 13.

29. Sony Labou Tansi, La Parenthèse de sang, Je, soussigné cardiaque, Paris, Éditions Hatier International, 1981, p. 16. 
30. Jean Chevalier, Alain Gheerbrant, Dictionnaire des symboles, Paris, Édition Robert Laffont et Jupiter, 1982, p. 952.

31. Louis-Vincent Thomas, La terre africaine et ses religions, Paris, Librairie Larousse, 1975, p. 257.

32. Louis-Vincent Thomas, Le cadarre, Éditions Complexe, 1980, p. 47.

33. Louis-Vincent Thomas, Cinq essais sur la mort africaine, Paris, Karthala, 2013, p. 104.

34. Ibid., p. 60-61.

35. Louis-Vincent Thomas, La mort africaine, Paris, Payot, 1982, p. 80.

36. Louis-Vincent Thomas, Le cadavre, Bruxelles, Éditions Complexe, 1980, p. 10.

37. Louis-Vincent Thomas, Rites de mort, Paris, Fayard, 1985, p. 14.

38. Sony Labou Tansi, Éd. Carnières-Morlanwelz, Éd. Lansman, 1995, p. 75.

39. Sony Labou Tansi, op. cit., 1973, p. 44-45.

40. Sony Labou Tansi, Cercueil de luxe. La peau cassée, Montreuil-sous-Bois, Éditions Théâtrales, 2006, p.

41. Sony Labou Tansi, op. cit., 1973, p. 40-41.

42. Sony Labou Tansi, Qui a mangé Madame D'Avoine Bergotha? Lansman, 1989, p. 97.

43. Ibid., p. 102.

44. Sony Labou Tansi, Qu’ils le disent, qu'elles le beuglent, Éd. Carnières-Morlanwelz, Éd. Lansman, 1995, p. 14.

45. Lansman, 1992, p. 93-97.

46. Sony Labou Tansi, op. cit., 1995, p. 38.

47. Sony Labou Tansi, op. cit., 1973, p. 74.

48. Ibid., p. 75-76.

49. Sony Labou Tansi, op. cit., 2006, p. 38.

50. Ibid., p. 54.

51. Ibid., p. 54.

52. Ibid., p. 54-55.

53. Sony Labou Tansi, op. cit., 1989, p. 49.

54. Sony Labou Tansi, op. cit., 1973, p. 13.

55. Sony Labou Tansi, op. cit., 1981, p. 45.

56. Ibid., p. 59.

57. Louis-Vincent Thomas, Mort et pouvoir, Paris, Payot, 1978, p. 9. 